Z. klin. Chem. u. klin. Biochem.

7. Jg., S. 384-389. Juli 1969

\title{
Elektrolyte und renale Enzymausscheidung
}

\author{
Von W. RaAB und M. Hohenegger \\ Alus dem Institut für mediainische Chemie (Vorstand: Prof. Dr. F. Seelicb) und aus dem Insticut für experimentelle Patbologie \\ (Vorstand: Prof. Dr. A. Lindner) der Universität Wien
}

(Eingegangen am 28. März 1969)

An 250 Ratten wurde der Einfluß von Natriumkarenz, Natriumbelastung, Kaliumkarenz und Kaliumbelastung auf drei aus der Niere stanmende Enzymaktivitäten des Harnes (,Lcucinaminopeptidase“, Lactatdehydrogenase, alkalische Phosphatase) untersucht. Im Gegensatz zum akuten Experiment über wenige Stunden bewirkt eine längerdauernde Belastung des Organismus mit Natrium oder Kalium keine Veränderung der renalen Enzymausscheidung. - Unter Bedingungen, die zur hỵpokealiämischer Nephrose führen, erfolgt - in gleicher Weise wie bei anderen Nephrosen - cin Anstieg der renalen Enzymuric. - Bei Natriumkarenz ließ sich ebenfalls ein Anstieg der drei untersuchten Harnenzymaktivitäten nachweisen, wobei aber das Zustandekommen dieses Effektes nicht ganz klar scheint.

\section{Electrolytes and renal excretion of encymes}

In 250 rats, the influence of sodium and potassium loading and sodium and potassium depletion on renal enzyme cxerction (,,leucine aminopeptidase", lactate dehydrogenase and alkaline phosphacase) was investigated. All threc urinary cnzymic activities derive from the kidney. In contrast to acute experiments, loading of the organism with sodium or potassium does not alter renal enzyme excretion over longer periods of time. As already well documented, in potassium depletion, kaliopenic nephropathy is provoked; the resulting changes in permeability of tubular cells produce an increase in urinary enzymic activities derived from the kidney. In sodium depletion increased activitics of urinary I.AP, LDH and $\lambda$ P were also encountered. The mechanism, by which sodium depletion might lead to increased renal enzyme excretion, however, remains to be explained.

Die Niere stellt neben dem Serum und bestimmten Anhangsdrüsen des Urogenitaltraktes die Hauptquelle der Harnenzyme dar; deshalb gehen die meisten Nierenerkrankungen mit Inderungen von Harnenzymaktivitäten einher. Auch geringe, morphologisch noch nicht faßbare Nierenteränderungen bewirken oft schon signifikante Steigerungen bestimmter Enzymaktivitäten im Harn. Eine Ubersicht über die bisher vorliegenden klinischen und experimentellen Befunde wurde bereits an anderer Stelle gegeben (1).

Nierenerkrankungen stehen in enger Beziehung zum Elektrolytstoffwechsel. Einerseits gewährleistet eine intakte Nierenfunktion die Aufrechterhaltung des Elektrolytgleichgewichtes im Organismus und andererseits führen bestimmte Elektrolytmangelsyndrome zu schweren Nierenschäden. Aus diesem Grund schien es von praktischer Bedcutung, dic Veränderungen der renalen Enzymausscheidung bei Elektrolytkarenz und Elektrolytbelastung zu untersuchen. Uber die bei kurzdauernder Ändcrung von Wasser- und Elektrolytausscheidung bei Mensch und Ratte einsetzenden Verschiebungen der renalen Enzymurie wurde bereits an anderer Stelle berichtet (2).

Die vorliegende Arbeit beschäftigt sich mit den bei längerdaucrnder Änderung der Elektrolytzufuhr ( $\mathrm{Na}$ triumkarenz, Natriumbclastung, Kaliumkarenz, Kaliumbclastung) auftretenden Veränderungen der renalen Enzymausscheidung. Untersucht wurden folgende drei aus der Niere stammenden Enzyme im Rattenharn: "Lcucinaminopeptidase", Lactatdehydrogenase und alkalische Phosphatase').

1) Enz)me: I.AP = I.cucinaminopeptidase (EC 3.4.1.2), 1.DH - Lactatdehydrogenase $=$ L-Lactat: NAD Oxydorcduktase (EC 1.1.1.27), $A P=$ Alkalische Phosphatase (EC 3.1.3.1).

\section{Versuche und Ergebnisse}

\section{Allgemeines}

Dic Untersuchungen crfolgten an Ratten, wobei der Tierstamm, das Geschlccht und das Körpergewicht der Versuchstiere bei jeder Scrie gesondert angeführt wird. Bei den langfristigen Versuchen wurden die Enzymaktivitäten jewreils in der 24-StundenHarnportion geprüft. Die Ratten wurden in Stoffwechselkāfigen (Plastikkäfige mit doppeltem Drahtmascheneinsatz) gehalten und crhielten am Beginn der 24-Stunden-Harnsammelperiode jeweils $10 \mathrm{~m} /$ Wasser mit der Schlundsonde verabreicht. Die Harnsammlung erfolgte unter Paraffin.

In früheren Untersuchungen konnte immer wieder gezcigt werden (vgl. 2,3,4), daß in verschiedenen Tierkollektiven in Abhängigkeit vom Alter, rom Geschlecht und rom Tierstamm große Divergenzen in den absoluten Werten der renalen Enzymausscheidung bestehen. Im Einzelkollektiv (gleiche Gewichtsklasse, gleicher Tierstamm, gleiches Geschlecht) liegen hingegen dic W'crte bei den einzelnen Versuchstieren innerhalb einer Streubrcite von $\pm 15 \%$.

Als Ausgangswert ("Normalwert") wurde dic mittlere Enzymbzw. Elcktrolytausschcidung von zwei aufeinanderfolgenden Tagen vor dem Versuchsbeginn angenommen.

Im Vergleich zu der Strcubrcitc der Enzymaktivitäten im 24Stunden-Harn unter Normalbedingungen (Ausgangswerte bzw. Kontrollwerte) ergab sich bei den Bclastungs- und Karenzversuchen eine höhere Strcuung der Einzelwerte. In kcinem Fall übcrstieg dic Strcubreite $(\vec{X} \pm 2 \sigma)$ bei den hier angeführten $V$ ersuchsscrien $33^{\circ} \%$ des Mlittelwertes, mit Ausnahme des Versuches „Kaliumkarenz", bei dem die Strcubreite gesondert in der Tabelle angeführt ist. Dic Berechnung der Streuung sowie der Signifikanz crfolgte nach dem $t$-Test.

Dic Bestimmung der Enzymaktivitäten erfolgte jeweils im zentrifugierten und durch 3 Stdn. gegen Hicßendes Leitungswasser dialysierten Harn; die Untersuchungen der "Leucinaminopeptidase "l), der Lactatdehydrogenasc') und der alkalischen Phosphatase $^{3}$ ) crfolgten mit den gleichen Methoden, wie sie früher beschrieben wurden (3). Die Aktivität der LAP und LDH wurde den internationalen Gepflogenheiten gemä $B$ in $\mathrm{mU}$ angegeben, die Aktivität der AP wurde in mMol-Einheiten nach BESSEY-LoowrY ${ }^{2}$ ) 2) $1 \mathrm{mMlol}$-Einheit nach Bessey-Lowry $=16,67 \mathrm{mU}$. 


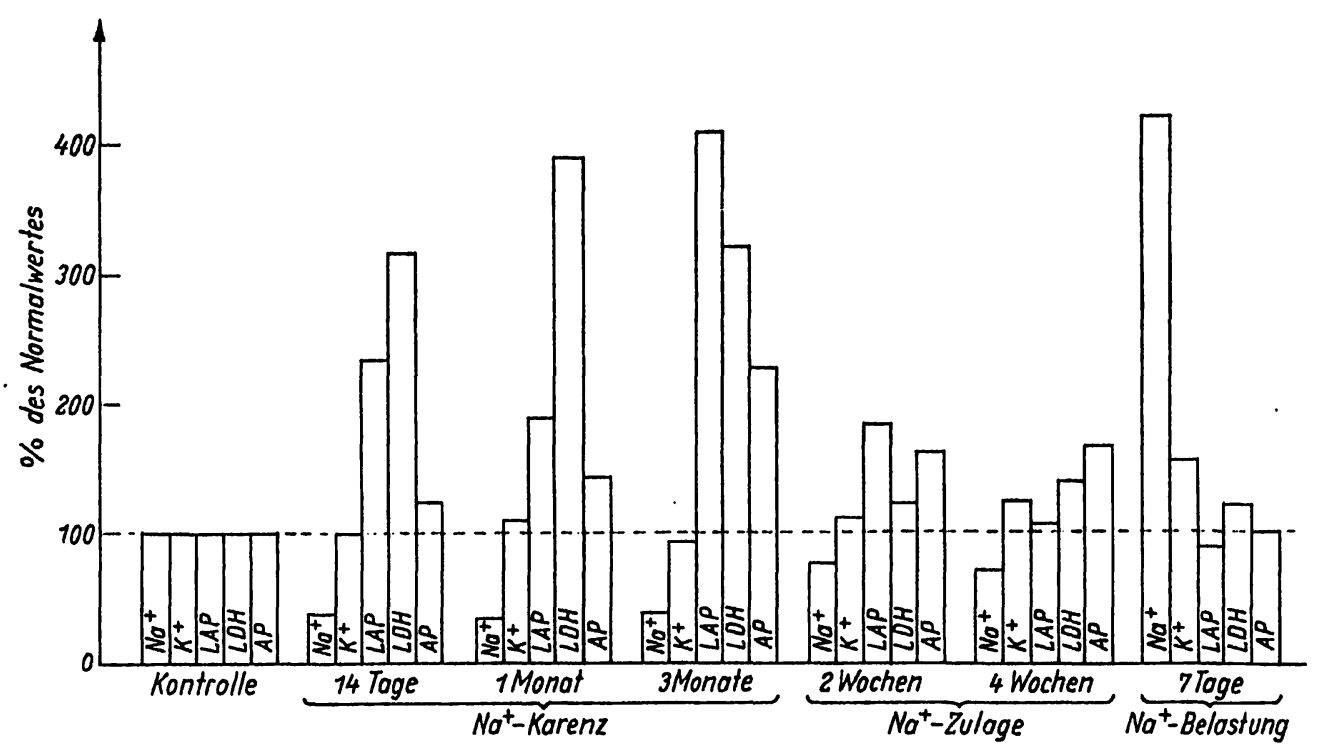

Abb. 1

Renale Enzymausscleidung und Elektrolyte itn Harn bei $\mathrm{Na}^{+-}$ Karenz, -Zulage und -Bclastung. Mittelwerte der Versuchsergebnisse bei 20 Ratten, wobci als Ausgangswert $(=100 \%)$ der Mittelwert aus Bestimmungen an 2 aufeinanderfolgenden Tagen vor Versuclisbeginn eingesetzt ist

bercehnet (Testkombinationen der Fa. Boehringer, Mannheim). Dic Bestimmung der Elektrolyte im Harn wurde mit Hilfe cines Flammenphotometers durchgeführt (4).

Natriumkarenz

\section{Serie $I$}

20 männliche, $240-260 \mathrm{~g}$ schwere Wistar-Ratten eigencr Zucht erhiclten durch 3 Monatc cinc Natrium-arme Ennährung, be-

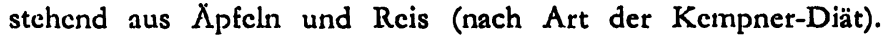
Leitungswasser wurde unverändert ad libitum gegeben. Aus der täglich verzehrten Menge an Reis und Apfeln errechnete sich eine tägliche Natriumaufnahme pro Tier von durchschnittlich $60 \mu \mathrm{Val}$. - Nach drcimonatiger Natrium-armer Ernährung erhielten dic Ticre durch 4 Wochen eine tägliche Natriumzulage von durchschnittlich $130 \mu \mathrm{Val}$. Anschließend erfolgte eine $\mathrm{Na}$ triumbelastung mit $750 \mu \mathrm{Val}$ pro Tag durch 7 Tage. Natrium wurde in Form ciner $\mathrm{NaCl}$-Lösung mit der Schlundsonde verabreicht.

Die Veränderungen der renalen Enzymurie und der Elektrolytausscheidung über den gesamten Versuchszeitraum sind in Tabelle 1 zusammengestellt. Mit sinkender Natriumzufuhr erfolgte eine Steigerung aller drei untersuchten Harnenzymaktivitäten. Eine genaue Korrelation mit der Dauer der Natrium-armen Ernährung ließ sich nicht nachweisen. Die Aktivitätszunahme erreichte bei einzelnen Enzymen das Drei- bis Vierfache des Normalwertes (Ausgangswert vor Versuchsbeginn, Mittelwert aus Bestimmungen an zwei aufeinanderfolgenden Tagen). - Nach Natriumzulage kehrten die Harnenzymaktivitäten langsam wieder zum Ausgangswert zurück. In Abbildung 1 ist das Verhalten von Natrium und Kalium im Harn sowie der drei Enzymaktivitäten im Harn über den gesamten Versuchszeitraum graphisch dargestellt. Betont werden muß, daß die Tiere der Serien I und II über den gesamten Versuchszeitraum ein gutes Gedeihen zeigten.

Dieser überraschende Befund eines Anstiegs von Harnenzymaktivitäten bei Natrium-armer Ernährung war Anlaß zur Durchführung ähnlicher Untersuchungsreihen.

\section{Serie $I I$}

Eine Gruppe von 20 Ratten (weibliche Tiere, Stamm FIW 49 Biberach, 280 - $320 \mathrm{~g}$ Körpergewicht) erhiclt wieder einc ReisApfel-Diät; 10 weitcrc Tiere bekamen die gleiche Diät mit NatriumZulage. Diese Tiere dienten als Kontrollen zum Ausschluß unspezifischer Effekte über den Versuchszcitraum von 9 Wochen. Dic tägliche Natriumaufnahme der Versuchsticre betrug im Anfang $80 \mu \mathrm{Val}$, später nach Gewöhnung der Ratten an dic Ernährungsform bis zu $140 \mu \mathrm{Val}$. Dic Kontrollticre nahmen pro Tag ctwa $800 \mu \mathrm{Val}$ Natrium auf.

Tab. 1

Renale Enzymurie, Elektrolyte im Harn und Harnvolumen bei Natriumkarenz, Natriumzulage und Natriumbelastung InME $=$ nıMol-Einheiten naclı Bessey-Lowry

\begin{tabular}{|c|c|c|c|c|c|c|c|}
\hline \multicolumn{2}{|c|}{$\begin{array}{l}\text { Versuchstiere } \\
\text { (20 RatteII) }\end{array}$} & $\begin{array}{c}\text { Harn } \\
\text { inl } 24 \text { Stun. }\end{array}$ & $\stackrel{\mathrm{Na}^{+}}{\mu \mathrm{Val} / 24 \mathrm{Stdn}}$ & $\stackrel{\mathrm{K}^{+}}{\mu \mathrm{Val} / 24 \mathrm{Stdn}}$ & $\begin{array}{c}\text { LAP } \\
\mathrm{mU} / 24 \text { Std }\end{array}$ & $\begin{array}{c}\text { LDH } \\
\mathrm{mu} / 24 \text { Stdn. }\end{array}$ & $\begin{array}{c}\text { AP } \\
\text { mME/24 Stdn. }\end{array}$ \\
\hline \multicolumn{2}{|c|}{$\begin{array}{l}\text { Kontrolle } \\
\text { (vor Versuchsbeginn) }\end{array}$} & 11 & $\begin{array}{c}196 \pm 25 \\
(100 \%)\end{array}$ & $\begin{array}{c}224 \pm 22 \\
(100 \%)\end{array}$ & $\begin{array}{c}20,4 \pm 1,9 \\
(100 \%)\end{array}$ & $\begin{array}{c}120 \pm 14 \\
(100 \%)\end{array}$ & $\begin{array}{c}21,6 \underset{1}{1} 2,8 \\
(100 \%)\end{array}$ \\
\hline Na-Karellz & $\begin{array}{l}14 \text { Tage } \\
1 \text { Monat } \\
2 \text { Monate } \\
3 \text { Monate }\end{array}$ & $\begin{array}{r}13 \\
9 \\
10 \\
8\end{array}$ & 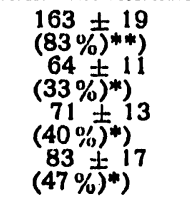 & $\begin{array}{c}231 \pm 30 \\
(103 \%) \\
248 \pm 32 \\
(110 \%) \\
235 \pm 24 \\
(105 \%) \\
201 \pm 25 \\
(90 \%)\end{array}$ & $\begin{array}{l}45,2 \pm 8,6 \\
\left.(220 \%)^{*}\right) \\
37,5 \pm 7,8 \\
\left.(190 \%)^{*}\right) \\
51,6 \frac{1}{\%} 11,3 \\
\left.(250 \%)^{*}\right) \\
82,2 \frac{1}{\%} 17,2 \\
\left.(410 \%)^{*}\right)\end{array}$ & $\begin{array}{l}382 \pm 71 \\
\left.(320 \%)^{*}\right) \\
461 \pm 90 \\
\left.(384 \%)^{*}\right) \\
372 \pm 52 \\
\left.(310 \%)^{*}\right) \\
378 \pm 49 \\
\left.(320 \%)^{*}\right)\end{array}$ & 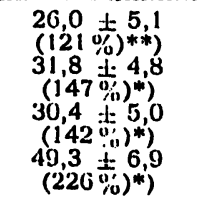 \\
\hline Na-Zulage & $\begin{array}{l}2 \text { Wochen } \\
4 \text { Wochen }\end{array}$ & $\begin{array}{l}12 \\
13\end{array}$ & $\begin{array}{l}155 \pm 25 \\
(79 \%) \\
133 \pm 14 \\
(68 \%)\end{array}$ & $\begin{array}{c}247 \pm 33 \\
(111 \%) \\
270 \pm 24 \\
(120 \%)\end{array}$ & $\begin{array}{c}36,5 \pm 9,1 \\
(185 \%) * 4) \\
23,2 \pm 6,3 \\
(116 \%)\end{array}$ & $\begin{array}{c}144 \frac{1}{15} \\
(120 \%) \\
130 \pm \frac{1}{130} 8 \\
(108 \%)\end{array}$ & 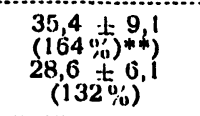 \\
\hline $\mathrm{Na}$-Belastun & 7 Tage & 8 & $\begin{array}{l}823 \\
(420 \%)^{*}\end{array}$ & $\begin{array}{c}347 \frac{1}{39} \\
\left.(154 \%)^{* *}\right)\end{array}$ & ${ }_{(81 \%)}^{16,2}$ & $\begin{array}{c}145 \pm 15 \\
(120 \%)\end{array}$ & $\begin{array}{c}22,5 \text { : } 2,9 \\
(104: 0)\end{array}$ \\
\hline
\end{tabular}

*) $P<0,001$ **) $P<0,01$ 
In Tabelle 2 sind die Ergebnisse der wöchentlichen bzw. vierzehntägigen Harnuntersuchungen zusammengestellt. Es fand sich der gleiche Effekt wie bei den Tieren der Serie I: bei längerer Natrium-armer Ernährung steigt die renale Enzymausscheidung an. In Abbildung $2 \mathrm{a}$ und $2 \mathrm{~b}$ sind die relativen Veränderungen von Elektrolyten und Enzymaktivitäten im Harn im Vergleich zur Kontrollgruppe graphisch dargestellt. Die Tiere der Serie II (Versuchstiere, Kontrolltiere) befanden sich über den gesamten Versuchszeitraum in gutem Zustand.

Wie aus Abbildung 2a deutlich hervorgeht, findet sich bei den Versuchstieren (Reis-Apfel-Diät) ein Anstieg der Kaliumausscheidung und eine Abnahme der Natriumausscheidung im Harn, während bei den Kontrolltieren (Reis-Apfel-Diät und Natriumzulage) die Natriumausscheidung ansteigt und die Kaliumausscheidung abnimmt.

Scrie III

8 Ratten (männliche Tiere, Stamm Sprague-Dawley eigener Zucht, Körpergewicht 180-240 g) erhielten eine völlig Natriumfreie Kost. Diese bestand aus praktisch Natrium-freien Würfelzucker und einem Vitaminpräparat (Protovit Roche), für dessen freundliche Uberlassung der Herstellerfirma herzlich gedankt sei. Das Trinkwasser der Tiere enthielt pro Liter $50 \mathrm{mVal} \mathrm{Kalium,}$ 2,5 mVal Calcium und 2,5 mVal Magnesium. Der Versuchszeitraum betrug 6 Wochen.

Die Ergebnisse der Harnuntersuchungen bei den Tieren der Serie III sind in Tabelle 3 zusammengestellt. Ergänzend wäre noch $z u$ bemerken, daß sich der regelmäßig kontrollierte Harn-pH-Wert über den Versuchzeitraum praktisch nicht veränderte (Schwankungen zwischen $\mathrm{pH}$ 6,5 und 7,0). Als $\mathrm{Maß}$ für den Allgemeinzustand dieser Natrium- und Protein-frei ernährten Tiere ist in der Tabelle jeweils auch das Körpergewicht angegeben. Präterminal fand sich eine Abnahme des Körgewichts bis auf $50 \%$ des Ausgangswertes. In den ersten 3 Tagen des Versuches verloren die Tiere durchschnittlich $1500 \mu$ Val Natrium, da im Harn ausgeschiedenes Natrium nun nicht mehr durch die Nahrung ersetzt werden konnte. Am 4. Versuchstag lag jedoch nur eine geringe Erhöhung einer der drei untersuchten Harnenzymaktivitäten (LAP) vor, während die beiden anderen Harnenzymaktivitäten (LDH und AP) eine Verminderung zeigten. Bei den Tieren der Serie III fällt besonders auf, daß bis zum 14. Tag des Versuches die Harn-

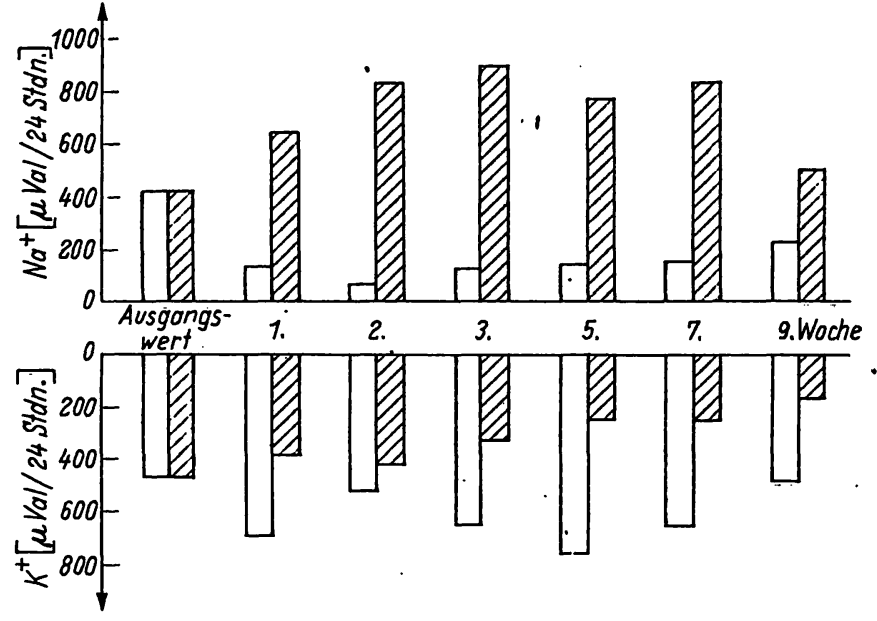

Abb. $2 a$

Elektrolyte im Harn bei Reis-Apfel-Diät mit und ohne Natriumzulage $\square$ Reis-Apfel-Diät

//// Reis-Apfel-Diät mit Natriumzulage

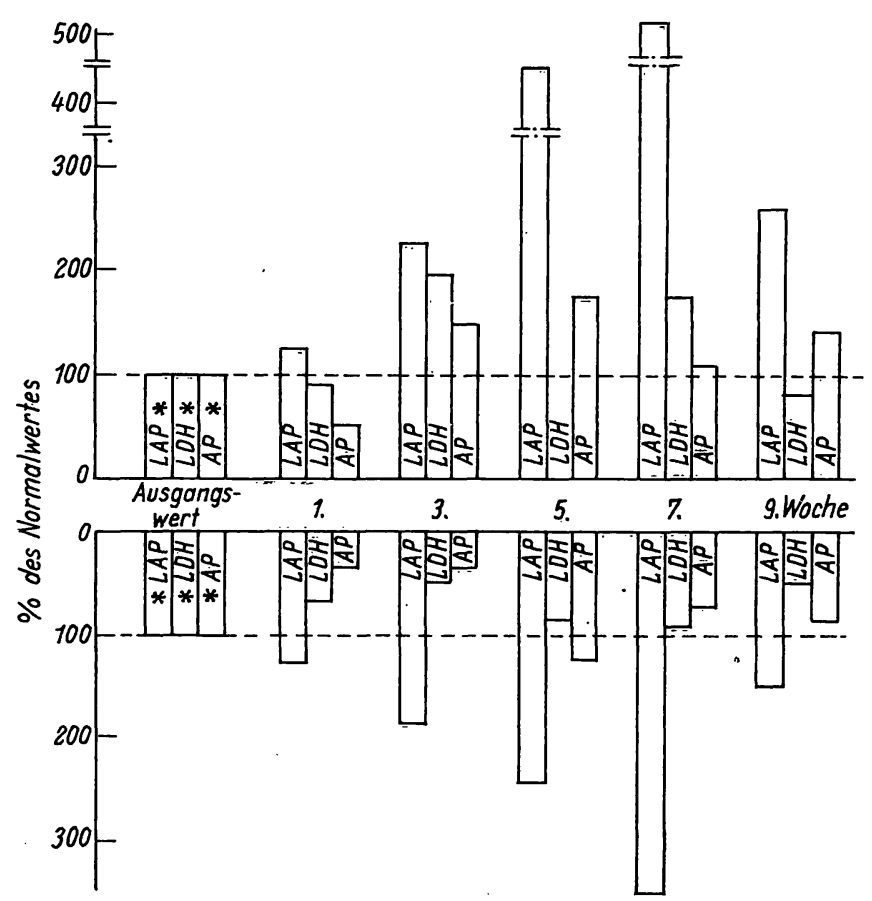

Abb. 2b

Harnenzymaktivitäten bei Reis-Apfel-Diät mit und ohne Natriumzulage. Reis-Apfel-Diät (Versuchstiere) oberer Teil. Reis-Apfel-Diät mit Natriumzulage (Kontrolltiere) unterer Teil

*) Mittelwerte des gesamten Kollektivs Versuchstiere und Kontrolltiere

Tab. 2

Renale Enzymurie und Elektrolyte im Harn bei Versuchstieren (V) unter Kempner-Diät und Kontrolltieren (K) unter gleicher Diät mit Natriumchloridzulage. $\mathrm{mME}=\mathrm{mMol}$-Einheiten nach Bessey-Lowry

\begin{tabular}{|c|c|c|c|c|c|c|}
\hline Zeitpunkt & & $\begin{array}{c}\text { Natrium } \\
\mu \mathrm{Val} / 24 \text { Stdn. }\end{array}$ & $\begin{array}{c}\text { Kalium } \\
\mu \mathrm{Val} / 24 \text { Stdn. }\end{array}$ & $\begin{array}{c}\text { LAP } \\
\text { mU/24 Stdn. }\end{array}$ & $\begin{array}{c}\mathrm{LDH} \\
\mathrm{mU} / 24 \text { Stdn. }\end{array}$ & $\frac{A P}{\text { mME/24 Stdn. }}$ \\
\hline Beginn & $\left.\begin{array}{l}\mathrm{v} \\
\mathrm{K}\end{array}\right\}$ & $417 \pm 39$ & $448 \pm 48$ & $11,0 \pm 1,3$ & $282 \pm 31$ & $16,6 \pm 2,4$ \\
\hline $\begin{array}{l}1 \text { Woche } \\
2 \text { Wochen } \\
3 \text { Wochen } \\
5 \text { Wochen } \\
7 \text { Wochen } \\
9 \text { Wochen }\end{array}$ & $\begin{array}{l}\text { V } \\
\text { K } \\
\text { V } \\
\text { K } \\
\text { V } \\
\text { K } \\
\text { V } \\
\text { K } \\
\text { V } \\
\text { K } \\
\text { V } \\
\text { K }\end{array}$ & $\begin{array}{l}157 \pm 29 *) \\
652 \pm 72 \\
\left.73 \pm 12^{*}\right) \\
830 \pm 90 \\
\left.142 \pm 27^{*}\right) \\
900 \pm 145 \\
160 \pm 28 *) \\
780 \pm 69 \\
\left.166 \pm 30^{*}\right) \\
840 \pm 88 \\
\left.250 \pm 37^{*}\right) \\
460 \pm 72\end{array}$ & $\begin{array}{l}670 \pm 81 \\
360 \pm 70 \\
514 \pm 77 \\
407 \pm 62 \\
636 \pm 94 \\
318 \pm 57 \\
762 \pm 112 \\
234 \pm 51 \\
654 \pm 107 \\
230 \pm 50 \\
480 \pm 102 \\
170 \pm 54\end{array}$ & $\begin{array}{l}14,0 \pm 1,4 \\
15,0 \pm 1,9 \\
17,5 \pm 2,8 \\
22,0 \pm 3,1 \\
\left.25,0 \pm 2,0^{*}\right) \\
20,1 \pm 3,2 \\
\left.49,0 \pm 8,3^{*}\right) \\
27,0 \pm 4,7 \\
\left.56,0 \pm 10,3^{*}\right) \\
39,0 \pm 5,1 \\
\left.29,4 \pm 4,6^{*}\right) \\
16,8 \pm 1,9^{2} .\end{array}$ & $\begin{array}{l}242 \pm 28 \\
185 \pm 16 \\
204 \pm 31 \\
165 \pm 32 \\
548 \pm 88 *) \\
130 \pm 21 \\
240 \pm 32 \\
220 \pm 38 \\
\left.480 \pm 91^{*}\right) \\
233 \pm 28 \\
200 \pm 30 \\
140 \pm 26\end{array}$ & $\begin{array}{c}7,5 \pm 1,3 \\
6,3 \pm 1,2 \\
\pm \\
25,4 \pm 6,0 \\
6,2 \pm 2,0 \\
29,6 \pm 4,8 *) \\
22,0 \pm 3,1 \\
18,0 \pm 4,3 \\
12,0 \pm 3,0 \\
23,3 \pm 4,0 *) \\
14,3 \pm 3,2\end{array}$ \\
\hline
\end{tabular}

*) $P<0,01$ 
Tab. 3

Elektrolyte im Harn, Körpergewicht und renale Enzymurie bei Ratten unter Natrium-freier Kost. $\mathrm{mME}=\mathrm{mMol}-\mathrm{Einh}$ eiten nach Bessey-Lowry

\begin{tabular}{|c|c|c|c|c|c|c|c|}
\hline Zeitpunkt & $n$ & $\underset{\mu \text { Val/24 Stdn. }}{\text { Natrium }}$ & $\begin{array}{c}\text { Kalium } \\
\mu \text { Val/24 Stdn. }\end{array}$ & $\begin{array}{c}\text { Körper- } \\
\text { gewicht (g) }\end{array}$ & $\begin{array}{c}\text { LAP } \\
\mathrm{mU} / 24 \text { Stdn. }\end{array}$ & $\begin{array}{c}\text { LDH } \\
\mathrm{mU} / 24 \mathrm{Stdn} .\end{array}$ & $\mathrm{AP}$ AP 24 Stdn. \\
\hline $\begin{array}{l}\text { Versuchs- } \\
\text { beginn }\end{array}$ & 8 & $\begin{array}{c}671 \pm 44 \\
(100 \%)\end{array}$ & $\begin{array}{c}325 \pm 24 \\
(100 \%)\end{array}$ & $\begin{array}{c}240 \pm 15 \\
(100 \%)\end{array}$ & $\begin{array}{l}15,2 \pm 1,3 \\
(100 \%)\end{array}$ & $\begin{array}{c}101 \pm 16 \\
(100 \%)\end{array}$ & $\begin{array}{c}12,6 \pm 2,4 \\
(100 \%)\end{array}$ \\
\hline $\begin{array}{l}\text { 4. Tag } \\
\text { 6. Tag } \\
\text { 12. Tag } \\
\text { 21. Tag } \\
\text { 28. Tag } \\
\text { 35. Tag }\end{array}$ & $\begin{array}{l}8 \\
8\end{array}$ & $\begin{array}{l}42 \pm 10 \\
\left.(6 \%)^{*}\right) \\
24 \pm 11 \\
\left.(3 \%)^{*}\right) \\
23 \pm \pm 9 \\
\left.(3 \%)^{*}\right) \\
31 \pm 14 \\
\left.(4 \%)^{*}\right) \\
16 \pm 7 \\
\left.(2 \%)^{*}\right) \\
5 \pm^{4} \\
\left.(1 \%)^{*}\right)\end{array}$ & $\begin{array}{c}252 \pm 28 \\
(74 \%)^{* *)} \\
198 \pm 34 \\
\left.(61 \%)^{*}\right) \\
238 \pm 41 \\
\left.(71 \%)^{*}\right) \\
313 \pm 44 \\
(95 \%) \\
165 \pm 29 \\
\left.(51 \%)^{*}\right) \\
342 \pm 72 \\
(106 \%)\end{array}$ & $\begin{array}{c}220 \pm 28 \\
(92 \%) \\
203 \pm 34 \\
(85 \%) \\
210 \pm 37 \\
(88 \%) \\
180 \pm 40 \\
(80 \%)^{* *)} \\
160 \pm 56 \\
\left.(67 \%)^{*}\right) \\
143 \pm 51 \\
\left.(60 \%)^{*}\right)\end{array}$ & $\begin{array}{c}18,7 \pm 2,8 \\
(127 \%) \\
5,3 \pm 2,2 \\
(35 \%) \\
11,0 \pm 3,1 \\
(73 \%) \\
17,6 \pm 2,8 \\
\left.(118 \%)^{*}\right) \\
18,5 \pm 3,4 \\
\left.(123 \%)^{*}\right) \\
24,8 \pm 8,9 \\
\left.(165 \%)^{*}\right)\end{array}$ & $\begin{array}{c}85 \pm 27 \\
(85 \%) \\
34 \pm 19 \\
\left.(34 \%)^{* *}\right) \\
44 \pm 21 \\
\left.(44 \%)^{* *}\right) \\
130 \pm 34 \\
\left.(130 \%)^{*}\right) \\
103 \pm 29 \\
\left.(103 \%)^{* *}\right) \\
110 \pm 35 \\
(110 \%)^{* *)}\end{array}$ & $\begin{array}{c}8,7 \pm 3,1 \\
(69 \%)^{* *)} \\
3,1 \pm 2,0 \\
\left.(26 \%)^{*}\right) \\
9,3 \pm 1,7 \\
(79 \%) \\
36,6 \pm 10,8 \\
(290 \%)^{*} \\
30,3 \pm 8,9 \\
(240 \%)^{*)} \\
34,5 \pm 12,5 \\
(270 \%)^{*)}\end{array}$ \\
\hline
\end{tabular}

*) $P<0,001 \quad$ **) $P<0,01$

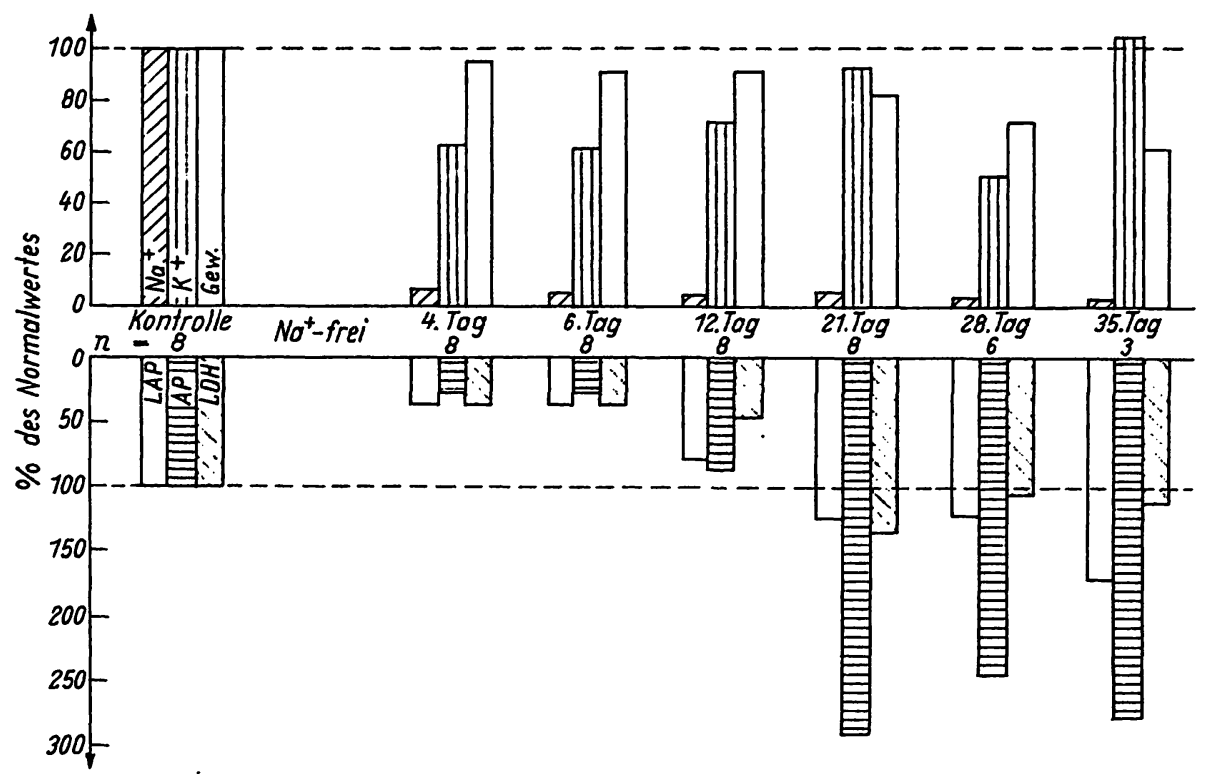

Abb. 3

Körpergewicht, Elektrolyte und Enzyme im Harn bei Natrium-freier Ernährung. Normalwert $=100 \%$

enzymaktivitäten eine Verringerung zeigten (mit Ausnahme der LAP am 4. Tag). Der auf Grund der Ergebnisse von Serie I und II erwartete Anstieg der renalen Enzymausscheidung trat erst in der dritten Versuchswoche ein; die LDH-Aktivität ließ nur geringen Anstieg erkennen. Abbildung 3 gibt eine graphische Darstellung der Ergebnisse von Serie III.

\section{Natriumbelastung}

In insgesamt sechs, Versuchsserien exhielten 110 Ratten verschiedenster Stämme, verschiedenster Gewichtsklassen und beider Geschlechter $0,15 \mathrm{M} \mathrm{NaCl}$-Lösung intraperitoneal 'verabreicht. Die Natriumbelastung variierte in den einzelnen Serien zwischen 0,31 und $1,55 \mathrm{mVal}$ pro Tier und Tag, die Gcsamtwerte lagen in den einzelnen Serien zwischen 0,93 und $21,7 \mathrm{mVal}$ pro Tier. Ferner ethiclten 20 Tiere (weibliche Ratten des Stammes FW 49 Biberach, Gewicht $280-310 \mathrm{~g}$ ) eine Woche lang täglich $10 \mathrm{ml}$ $0,15 \mathrm{M} \mathrm{NaCl}-\mathrm{Lösung}$ mit der Schlundsonde verabreicht.

In keiner der Serien konnte eine Veränderung von Harnenzymaktivitäten als Folge der Natriumbelastungen festgestellt werden.

\section{Kaliumkarenz}

Die Tierversuche, in denen eine Erhöhung der renalen Enzymausscheidung als Folge eines Kaliummangels festgestellt werden konnte, wurden bereits an anderer
Stelle publiziert (4). Der Vollständigkeit halber soll aber im folgenden eine kurze Wiederholung der Versuchsmethode und der Ergebnisse erfolgen.

40 Ratten erhielten täglich mit der Schlundsonde eine Aufschwemmung von $4 \mathrm{~g}$ Natriumpolystyrolsulfonat (Resonium $\mathrm{A}^{3}$ ), .Ionenaustauscherharz, welches Kalium aufnimmt und Natrium freisetzt) in $10 \mathrm{~m} /$ Wasser verabfolgt. Außerdem war das Trockenfutter der Tiere mit 4\% Natriumpolystyrolsulfonat versetzt. Nach dreiwöchiger Kaliumkarenz erfolgte eine Kaliumrestitution durch eine Woche ( $1 \mathrm{mVal}$ pro Tier pro Tag).

Wie in Tabelle 4 zusammengestellt, nahm unter der Behandlung mit Natriumpolystyrolsulfonat das Kalium der Leber auf 66\% des Ausgangswertes ab; die Kaliumausscheidung im Harn sank auf 27\% des Ausgangswertes. Die Natriumausscheidung im Harn stieg auf das Dreifache des Ausgangswertes an. Gleichzeitig erfolgte ein signifikanter Anstieg der renalen Enzymausscheidung. Eine bioptische Untersuchung der Niẹren von 10 Versuchstieren nach dreiwöchigem Kaliummangel erbrachte keine sicheren pathologischen Befunde (einzelne Areale mit trüber Schwellung). - Nach siebentägiger Kaliumrestitution trat eine rasche Normalisierung der Elektrolyte und Enzyme im Harn ein. Die Versuchsergebnisse sind in Abbildung 4 graphisch dargestellt.

3) Herstèller: WINTHROP Lab. 
Tab. 4

Kaliumgehalt der Leber, renale Enzymausscheidung und Elektrolyte im Harn bei Kaliumkarenz und Kaliumrestitution. $\mathrm{mME}=\mathrm{mMol}-\mathrm{Einh}$ eiten nach Bessey-Lowry

\begin{tabular}{|c|c|c|c|c|c|c|c|}
\hline Versuchstiere & $\begin{array}{l}\mathrm{K} \text { in Leber } \\
\mathrm{mVal} / 100 \mathrm{~g}\end{array}$ & $\begin{array}{c}\text { Harn } \\
\text { ml } 24 \text { Stdn. }\end{array}$ & $\underset{\mu \mathrm{Val}}{\mathrm{K}}$ & $\underset{\mu \mathrm{Val}}{\mathrm{Na}}$ & Werte im 24 LAP & $\underset{\mathrm{mU}}{\mathrm{LDH}}$ & $\underset{\mathrm{mME}}{\mathrm{AP}}$ \\
\hline $\begin{array}{l}\text { Ausgangswerte } \\
\text { (40 Tiere) }\end{array}$ & $33 \pm 6,4$ & 12 & 522 & 342 & $58 \pm 11$ & $117 \pm 23$ & $9,7 \pm 2,1$ \\
\hline $\begin{array}{l}\text { Kaliumkarenz } \\
7 \text { Tage }\end{array}$ & - & 10 & $\begin{array}{l}260 *) \\
(50 \%)\end{array}$ & $\begin{array}{c}514 \\
(150 \%)\end{array}$ & \multirow{3}{*}{$\begin{array}{c}73 \pm 16 *) \\
(126 \%) \\
82 \pm 24 *) \\
(141 \%) \\
93 \pm 22 *) \\
(160 \%)\end{array}$} & \multirow{3}{*}{$\begin{array}{c}\left.186 \pm 52^{*}\right) \\
(159 \%) \\
\left.280 \pm 101^{*}\right) \\
(240 \%) \\
543 \pm 210 *) \\
(464 \%)\end{array}$} & \multirow{3}{*}{$\begin{array}{c}\left.16,9 \pm 5,7^{*}\right) \\
(174 \%) \\
23,6 \pm 6,1 *) \\
(243 \%) \\
25,9 \pm 4,9 *) \\
(267 \%)\end{array}$} \\
\hline 14 Tage & - & 11 & - & - & & & \\
\hline 21 Tage & 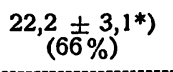 & 11 & $\begin{array}{l}143 *) \\
(27 \%)\end{array}$ & $\begin{array}{c}1030 *) \\
(305 \%)\end{array}$ & & & \\
\hline $\begin{array}{l}\text { Kaliumrestitution } \\
7 \text { Tage }\end{array}$ & $\begin{array}{c}26,6 \pm 2,6 *) \\
(81 \%)\end{array}$ & 13 & $\begin{array}{l}420 \\
(80 \%)\end{array}$ & $\begin{array}{c}401 \\
(117 \%)\end{array}$ & $\begin{array}{c}56 \pm 14 \\
(79 \%)\end{array}$ & $\begin{array}{l}96 \pm 14 \\
(82 \%)\end{array}$ & $\begin{array}{c}9,3 \pm 2,8 \\
(96 \%)\end{array}$ \\
\hline
\end{tabular}

*) $\mathrm{P}<0,005$

Tab. 5

Renale Enzymurie, Elektrolyte im Harn und Harnvolumen bei Kaliumbelastung (10 mVal $/ \mathrm{kg}) . \mathrm{mME}=\mathrm{mMol}$-Einheiten nach Bessey-Lowry

\begin{tabular}{|c|c|c|c|c|c|c|}
\hline Versuchstiere & $\begin{array}{c}\text { Harn } \\
\mathrm{m} l 24 \text { Stdn. }\end{array}$ & $\begin{array}{c}\mathrm{K}^{+} \\
\mu \mathrm{Val} / 24 \mathrm{Stdn}\end{array}$ & $\begin{array}{c}\mathrm{Na}^{+} \\
\mu \mathrm{Val} / 24 \mathrm{Stdn}\end{array}$ & $\begin{array}{c}\text { LAP } \\
\mathrm{mU} / 24 \mathrm{Stdn} .\end{array}$ & $\begin{array}{c}\mathrm{LDH} \\
\mathrm{mU} / 24 \mathrm{Stdn} .\end{array}$ & $\underset{\mathrm{mME} / 24}{\mathrm{AP}}$ Stdn. \\
\hline Kontrollen & 12 & $333 \pm 21$ & $182+21$ & $15,2 \pm 1,1$ & $121 \pm 16$ & $19,7 \pm 2,3$ \\
\hline $\begin{array}{l}\text { 1. Tag : } \\
\text { 2. Tag } \\
\text { 7. Tag }\end{array}$ & $\begin{array}{c}14 \\
(116 \%) \\
9 \\
(75 \%) \\
12 \\
(100 \%)\end{array}$ & $\begin{array}{c}\left.1800 \pm 310^{*}\right) \\
(540 \%) \\
\left.1850 \pm 240^{*}\right) \\
(550 \%) \\
\left.2563 \pm 506^{*}\right) \\
(770 \%)\end{array}$ & $\begin{array}{c}\left.504 \pm 41^{*}\right) \\
(278 \%) \\
\left.700 \pm 91^{*}\right) \\
(384 \%) \\
\left.1126 \pm 312^{*}\right) \\
(620 \%)\end{array}$ & $\begin{array}{c}18,5 \pm 1,4 \\
(122 \%) \\
17,7 \pm 2,0 \\
(117 \%) \\
14,5 \pm 2,1 \\
(96 \%)\end{array}$ & $\begin{array}{c}154 \pm 20 \\
(127 \%) \\
131 \pm 19 \\
(108 \%) \\
115 \pm 24 \\
(95 \%)\end{array}$ & $\begin{array}{c}17,9 \pm 4,1 \\
(91 \%) \\
19,5 \pm 5,3 \\
(99 \%) \\
17,5 \pm 3,4 \\
(89 \%)\end{array}$ \\
\hline
\end{tabular}

*) $\mathrm{P}<0,001$

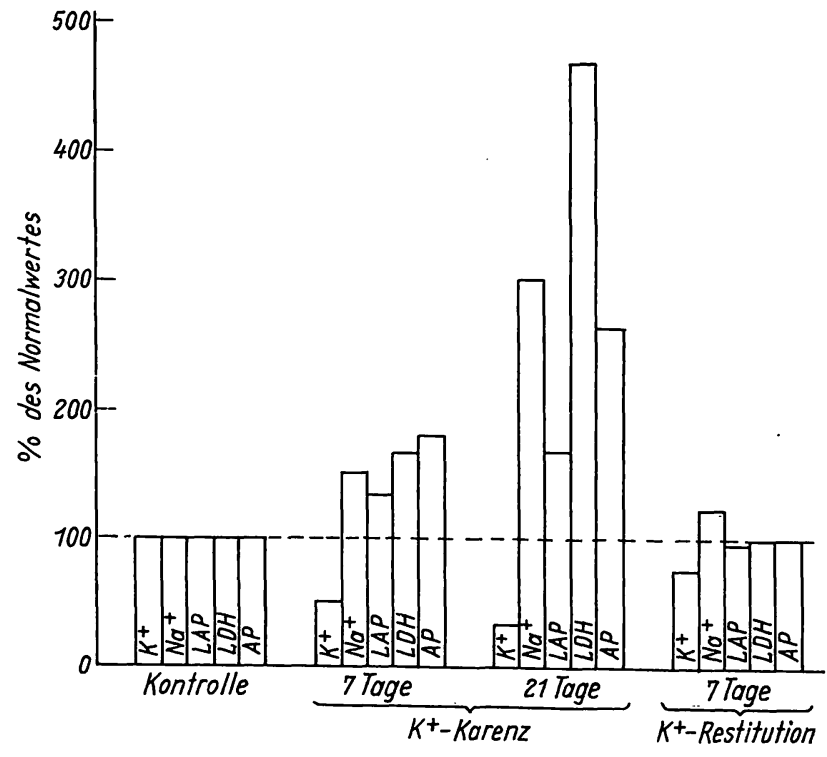

Abb. 4

Renale Enzymausscheidung und Elektrolyte im Harn bei $\mathrm{K}^{+-}$Karenz und $\mathrm{K}^{+}$-Restitution

\section{Kaliumbelastung}

20 Ratten (Wistar eigener Zucht, männliche Tiere, 210-230 g Körpergewicht) erhielten durch sieben Tage täglich $10 \mathrm{mVal}$ Kalium in $10 \mathrm{~m} l$ Wasser mit der Schlundsonde verabreicht. Das Kalium wurde zur Hälfte als Chlorid, zu einem Viertel als Bicarbonat und zu einem Viertel als Citrat gegeben. Am 1., 2. und 8. Tag des Versuches wurden Elektrolyte, Harn-pH und Harnenzyme bestimmt.

Die Ergebnisse finden sich in Tabelle 5 zusammengestellt. Nach einem kurzdauernden geringen Anstieg des Harnvolumens und zweier Harnenzymaktivitäten (LAP, LDH) am ersten Tag der Kaliumbelastung (vgl. akutes Experiment), blieben die Enzymwerte über den ge-

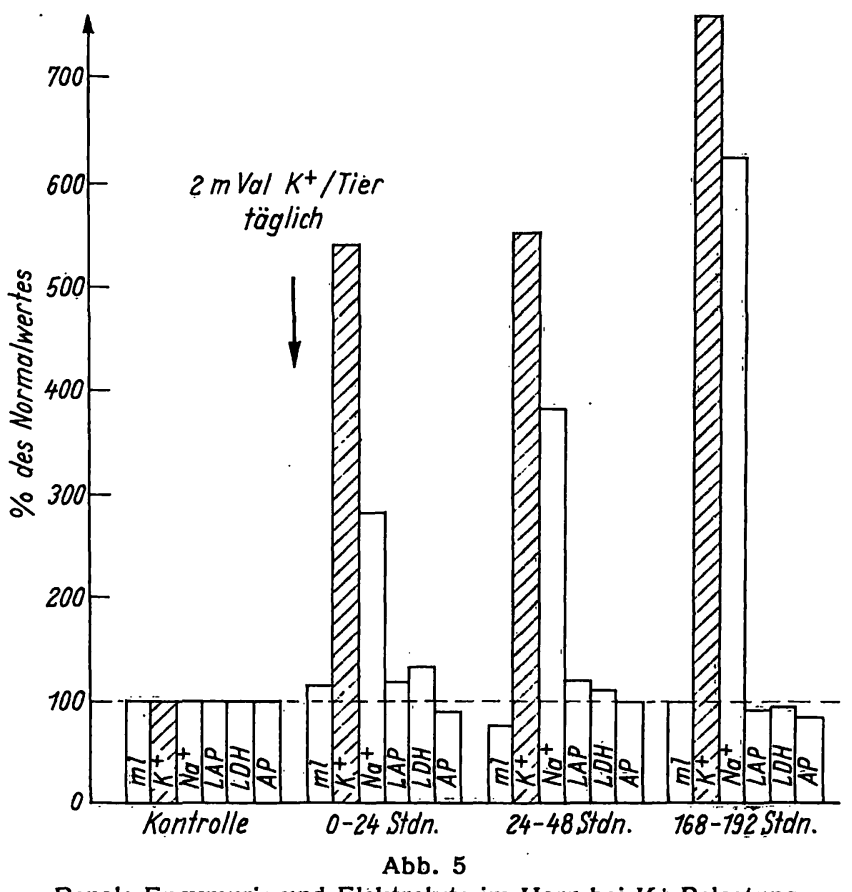

Renale Enzymurie und Elektrolyte im Harn bei $\mathrm{K}^{+-}$-Belastung

samten Versuchszeitraum praktisch unverändert. Die Kaliumausscheidung im Harn stieg als Folge der Kaliumbelastung auf das 7,7-fache des Kontrollwertes an. In Abbildung 5 sind die Versuchsergebnisse graphisch dargestellt.

\section{Diskussion}

Die vorliegenden Ergebnisse zeigen, daß weder eine Belastung mit Natrium noch eine Belastung mit Kalium zu Veränderungen der renalen Enzymausscheidung im 24-Stdn.-Harn führt; die drei unitersuchten Harnenzymaktivitäten LAP, LDH und AP blieben praktisch unverändert. 
Bei erhöhtem Angebot vermindert sich die tubuläre Reabsorption von Natrium aus dem Primärharn. Bei Kaliumbelastung wird Kalium vermehrt durch tubuläre - wahrscheinlich passive - Sekretion ausgeschieden (5). Wie die vorliegenden Untersuchungen gezeigt haben, bewirkt keine dieser funktionellen Veränderungen Verschiebungen der renalen Enzymausscheidung und läßt also jene Vorgänge in der Tubuluszelle unbeeinflußt, die für den Ubertritt von Enzymen in den Harn verantwortlich sind (Permeabilitätsprozesse, Zellerneuerung). Bei kurzfristiger Änderung der Natrium- und Kaliumausscheidung (Beobachtungszeitraum wenige Stunden) erfolgen jedoch Verschiebungen der renalen Enzymurie (2). Es sei aber an dieser Stelle ganz kurz auf die Tatsache hingewiesen, daß kürzere Beobachtungsperioden in der Regel wesentlich inkonstantere Werte der renalen Enzymausscheidung ergeben und durch Diurese oder Antidiurese leicht Änderungen vortäuschen können $(6,7)$.

Die Kaliumausscheidung bei Natrium-armer oder Natrium-freier Diät zeigt in den einzelnen Versuchsserien ein uneinheitliches Verhalten. Es ist anzunehmen, $\mathrm{da} ß$ hier eine weitgehende Abhängigkeit von der $\mathrm{Ka}$ liumzufuhr von außen besteht und die Veränderung des Natriumstoffwechsels für die Kaliumausscheidung nicht von entscheidender Bedeutung ist. Diese hier angeführten Befunde stehen in guter Úbereinstimmung mit verschiedenen Angaben in der Literatur, wonach sich beim Menschen ebenso wie im Tierexperiment die Kaliumausscheidung bei Veränderungen des Natriumhaushaltes uneinheitlich verhält $(8,9)$.

Aus den bisher vorliegenden Beobachtungen über die renale Enzymurie heraus ist der Befund der fehlenden Beeinflussung von Harnenżymaktivitäten bei Natriumbelastung nicht weiter überraschend. Anders ist dies hingegen bei Kaliumbelastung, bei der es zu einer gesteigerten tubulären Sekretionstätigkeit kommt; bei gesteigerter tubulärer Sekretion wurde bereits unter verschiedensten Bedingungen (Penicillin, Phenolrot u. a.) ein Anstieg der renalen Enzymausscheidung bei Mensch und Tier beobachtet $(3,10,11,12)$.
Die Steigerung der renalen Enzymausscheidung bei Kaliummangel ist leicht $\mathrm{zu}$ interpretieren; wie bei allen nephrotischen Nierenveränderungen kommt es auch bei hypokaliämischer Nephrose (vgl. 5, 13, 14, 15, 16, 17, $18,19)$ zu einer Schädigung der Tubulusepithelien. Damit vergesellschaftete Permeabilitätsveränderungen an den Bürstensäumen dürften einen vermehrten Übertritt von Enzymen in die tubuläre Flüssigkeit und damit in den Harn zur Folge haben („Enzymverlust"). Zu betonen ist bei diesen Versuchen das Ausmaß der biochemisch faßbaren Veränderungen zu einem Zeitpunkt, zu dem das morphologische Bild fast völlig unverändert ist.

Der in allen Versuchsserien nachweisbare Anstieg der renalen Enzymausscheidung bei Natriumkarenz kann heute noch nicht zufriedenstellend erklärt werden, ebensowenig wie die Wirkung einer Reis-Apfel-Diät mit Natriumzulage. Wesentlich ist die Feststellung, daß die Steigerung der Harnenzymaktivitäten nicht sofort, sondern erst nach zwei Wochen dauernder Natriumkarenz einsetzt, also zu einem Zeitpunkt, zu dem die Periode des initialen Natriumverlustes (vgl. Serie III) *vorüber ist und sich die Niere bereits längst an die Bedingungen der verminderten Natriumzufuhr angepaßt hat. Der initiale Natriumverlust ist daher ebensowenig unmittelbar Ursache für den Anstieg der renalen Enzymurie wie die gesteigerte tubuläre Reabsorption von Natrium.

Bei Annahme einer erhöhten Permeabilität der Tubulusepithelien als Ursache für die vermehrte Enzymurie bei Natriumkarenz bleibt die Frage nach der eigentlichen zugrunde liegenden Störung unbeantwortet. Bei den Tieren der Versuchsserie III (Natrium- und Eiweißfreie Ernährung) hat sicherlich auch die einsetzende Kachexie zur Steigerung der drei untersuchten Harnenzymaktivitäten beigetragen. Auffallend war aber hier die vergleichsweise nur sehr geringe Aktivitätszunahme der LDH im Harn.

Abschließend sei noch erwähnt, daß bisher nur eine Form einer Beeinflussung der Diuresebedingungen bekannt ist, die zu signifikanten Änderungen der renalen Enzymurie im Langzeitversuch führt - die Verabreichung von Hypophysenhinterlappenextrakt (20).

\section{Literatur}

1. RAAB, W., Enzymes and isoenzymes in urine. Actual problems in clinical Biochemistry, H. Huber, Bern, 2, 17 (1968). - 2. Hohenegger, M. und W. RAAB, Wien. $Z$. innere Med. (im Druck). 3. RaAb, W. und M. Hohenegger, Clin. Chim. Acta Amsterdam 20, 95 (1968). - 4. RAAB, W. und M. HOHÉNEGGER, Wien. klin. Wschr. 80, 402 (1968). - 5. GreBrsch, G. und G. MaLNIC, Grundlagen des renalen Kaliumtransportes. VI. Symp. Deutsch. Gesellsch. Nephrologie, Wien, 1968. Verlag d. med. Akad. Wien (im Druck). - 6. Hohenegger, M. und E. KAISER, Klin. Wschr. 45, 1252 (1967). - 7. Jösch, W. und U. C. Dubach, Clin. Chim. Acta Amsterdam 15, 325 (1967). - 8. PAPPER, S., L. SAXon, J. D. Rosenbaum und $\mathrm{H}$. W. Cohen, J. Laborat. Clin. Med. S. Louis 47, 776-782 (1956). - 9. Wiggins, W., C. H. Maury, R. H. Lyons und R. F. Pitrs, Circulation 3, 275 (1951). - 10. Ber GmanN, H. und G. F. Klostermann, Arch. klin. exp. Dermat. 218, 603
(1964). - 11. Bergmann, H. und F. Scheler, Klin. Wschr. 42, 275 (1964). - 12. Bergmann, H. und F. Truss, Med. Welt 1964, 1760. - 13. Antorne, B., D. Patte und R. Barcelo, Conséquences rénales du manque de potassium. Actualités nephrolog. de l'hôpital Necker. p. 67 Flammarion, Paris (1962). - 14. Biava, C..G., I. Dyrda, J. Genest und S. A. Benscome, Laborat. Invest. 12, 443 (1963). - 15. MiLne, M. D., R. C. MuehrCke und B. E. Heard, Brit. med. Bull. 23, 15 (1957). - 16. Muller, A. F., R. Veyrat und A. Grandchamp, Klin. Wschr. 46, 1241 (1968). 17. Oliver, J., M. MacDowell, L. G. Welt, M. A. Holliday, W. Hollander und W. E. Segar, J. exp. Med. 106, 563 (1957). 18. Relman, A. S. und W. B. Schwartz, New Engl. J. Med. 225, 195 (1956). - 19. Schwartz, W. B. und A. S. Relman, J. clin. Invest. 32, 258 (1953). - 20. RAAB, W., Wien. klin. Wschr. (im Druck).

Univ.-Doz. Dr. W. Raab A-1090, Wien, Österreich Währingerstraße 10 